\title{
A Red Flag in Sarcoidosis: Laryngeal Obstruction
}

\author{
Anne-Kathrin Brill ${ }^{a}$ Eberhard Seifert ${ }^{b}$ Thomas Geiser ${ }^{a}$ Sebastian R. Ott ${ }^{a}$ \\ ${ }^{a}$ Department of Pulmonary Medicine and ${ }^{b}$ Division of Phoniatrics, Department of Otorhinolaryngology, \\ Head and Neck Surgery, University Hospital and University of Bern, Bern, Switzerland
}

A 47-year-old Ethiopian with known biopsy-proven mucocutaneous facial sarcoidosis presented with dyspnea which had been rapidly progressive over a few weeks, hoarseness and stridor. Examination revealed a biphasic stridor; pulmonary function testing showed marked limitation of in- and expiratory flow rates and $\mathrm{FEV}_{1}$ was reduced to 2.19 liters (58\% of predicted value). Empey index [1] was $12.3\left[\mathrm{FEV}_{1}(\mathrm{ml}) / \mathrm{PEF}(\mathrm{l} / \mathrm{min})\right]$ consistent with fixed upper airway obstruction (fig. 1a). Further investigation of the larynx revealed circular narrowing (fig. $1 \mathrm{~b}$ ) and severe sarcoid involvement with a remaining dorsal glottis of $5 \mathrm{~mm}$ only (fig. 1c). The left vocal cord was fixed at paramedian position, and the right vocal cord moved at respiration only to intermediate position. During phonation the glottic closure was complete. Inspection of the proximal trachea ruled out any relevant stenosis of the upper and middle trachea. To avoid tracheotomy, an aggressive systemic anti-inflammatory therapy with prednisone (starting dose $1 \mathrm{mg} / \mathrm{kg} /$ day for 2 months, followed by stepwise reduction to a maintenance dose of $5 \mathrm{mg} /$ day) and azathioprine was started. Clinical symptoms improved after a few weeks and lung function (fig. 1d) significantly after 8 months. Laser treatment and/or dilatation and stent implantation would have been valuable treatment options in case of progressive dyspnea, but with these measures there is always the risk of irreversible damage to the vocal cords, potentially causing permanent changes in vocal tone and phonation. Today, after 18 months of treatment the patient is doing well and $\mathrm{FEV}_{1}$ remains stable in the range of $2.9-3$ liters $(78-80 \%$ of predicted value).

Laryngeal involvement in sarcoidosis causing upper airway obstruction is rare but potentially life-threatening. Thus, new onset dyspnea, stridor and typical lung functional limitations should be considered as a red flag in sarcoidosis as treatment options need to be evaluated immediately [2] to prevent tracheotomy.

\section{Financial Disclosure and Conflicts of Interest}

None of the authors has a financial relationship with a commercial entity that has an interest in the subject of this paper.

References

Anne-Kathrin Brill, MD

Department of Pulmonary Medicine

University Hospital and University of Bern

$\mathrm{CH}-3010$ Bern (Switzerland)

Tel. +41 31632 3490, E-Mail anne-kathrin.brill@insel.ch 

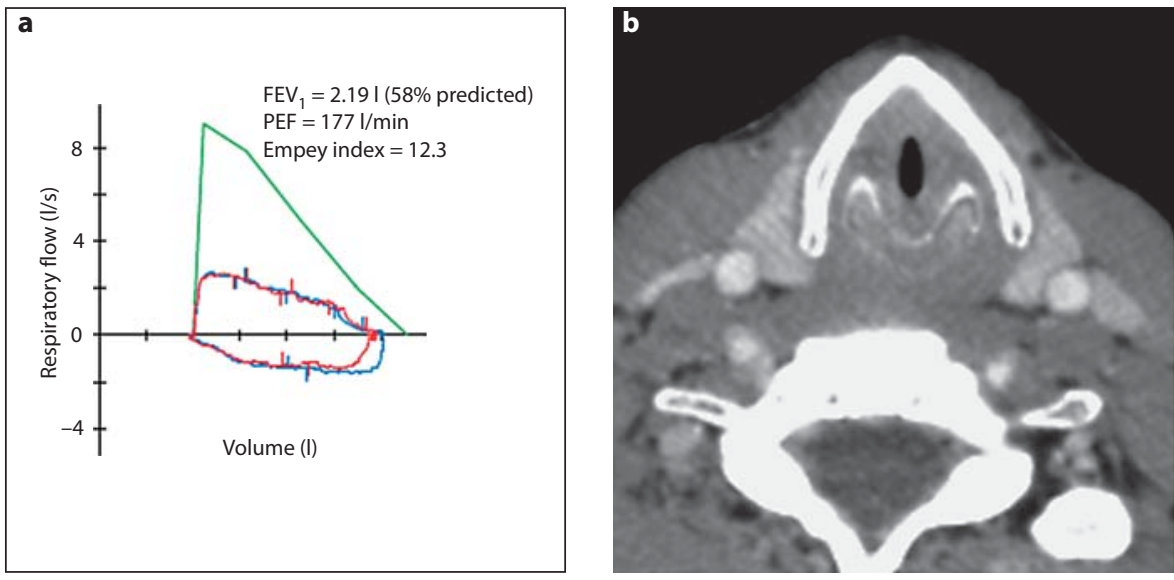

Fig. 1. a Initial flow volume curve, each curve representing one breath; positive flow indicates expiration. The Empey index of $>10$ indicates severe upper airway obstruction. b Computed tomography of the neck. c View of aryepiglottic folds before treatment. d Improvement of the flow volume curve and normalization of the Empey index after 8 months of treatment with prednisolone and azathioprine.
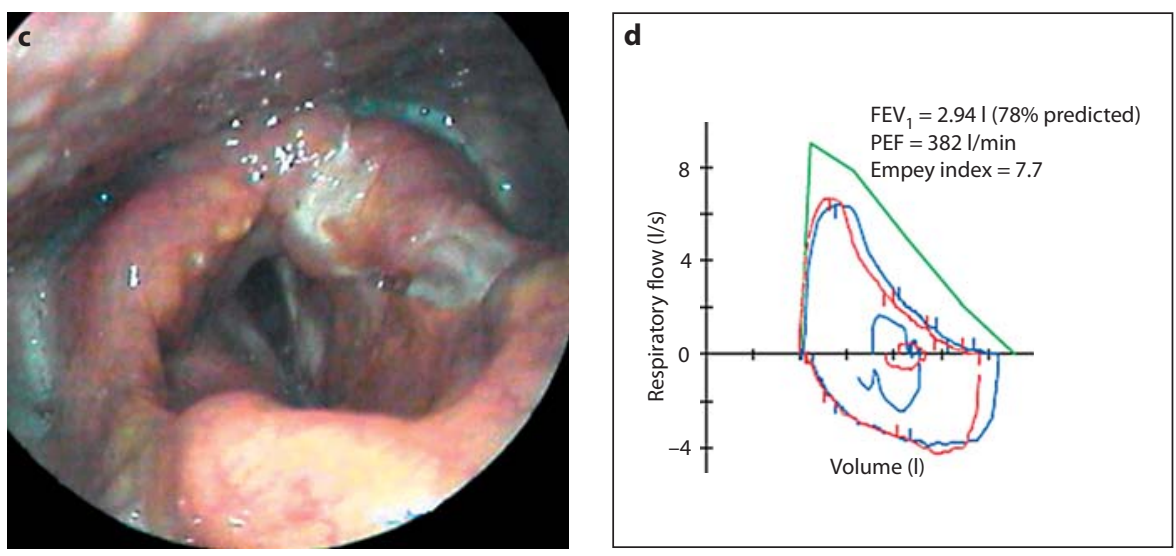